\title{
CULTURAL EVOLUTION, DESIGN AND PHILOSOPHY
}

\author{
C. VINIEGRA \\ Law School, Universidad Panamericana, Mexico.
}

\begin{abstract}
Human culture is the accumulation and evolution of results produced by countless design exercises. However, the process from which such design emerges lacks a solid scientific theory. Long-term sustainability issues force us to discover new boundaries and innovative design possibilities. This analysis makes reference to life's biological laws and the biophysical boundaries to which all living beings are constrained in order to propose a theoretical model that links restriction and capacity to four evolutionary strategies (Status Quo, Survival, Transformation, and Growth). The latter is related to cultural evolution, which identifies human culture as a phenomenon that stays within life's general evolution. The proposed model links evolutionary strategies to four distinct design goals within reach of human beings and to the fundamental philosophical building blocks that are consistent with each option of the model. After the model is presented, a statistical analysis is used for validation of the model. Despite great diversity of cultural and historical backgrounds in present societies, data form economic, energy and technological features of more than 100 countries show striking regularities related to the way that physical and social resources are applied to the process of economic and human development, and show consistency with the proposed design goals of the model. Among the most striking features discovered by this analysis it is worth noticing that, apparently, there is a minimal threshold of energy expenditure and research and development (R\&D) investment required to achieve a high level of social development. Furthermore, in order to achieve a positive and strong cooperative effect between energy and R\&D, worldwide evolution of such indexes seems to indicate that economic and social evolution is constrained to similar scaling laws, than those discovered for biological species. Although boundaries for cultural design are identified throughout this work, the evidence also suggests new design possibilities for moving forward into a transformational future supported by social technologies of metabolic (constructal) design.

Keywords: constructal, cultural evolution, design, economics, philosophy.
\end{abstract}

\section{INTRODUCTION}

While the modern era has been a time of advances of every kind, human beings still face the persistence of ancient problems such as curable diseases, social marginalization and wars. At the same time, human kind faces new challenges because of the deterioration of the environment, changes in climate conditions of the planet, exhaustion of key resources such as farming fields and clean water, along with the growth of social pathologies such as addictions and new violence phenomena that range from the family to the work environment. When thinking about old and new issues, decades of strategies aimed to solve them have accumulated and results are opposite of what it was expected while the provision of ideas seems insufficient to explain or solve even one important issue at its root.

Due to the recurrence of crisis of all types and to the probable perspective of disasters, such as a global climate change that can create among other things hunger and massive migration of people, it has become necessary to review the way in which we design human culture.

For the time being, there is not a wide consensus on what can happen or what should be done to avoid any negative scenario. Moreover, expectations range between apathy linked to overflowing optimism and anguish for perspectives that could generate the end of the human civilization. This means that decisions made on the short term, will have a high level of uncertainty and debate. 


\section{ASSUMPTIONS AND WORKING QUESTIONS}

\subsection{Assumptions}

1. The human being is circumscribed to the biological rules that make life possible.

2. There is a possibility of a significant change in culture design (change of era) due to the exhaustion of designs upon which modern culture has been built.

3. Sensory experience makes possible the creation of concepts over which human cognition is built.

4. The environmental scenario is adverse in the future.

5. Humans are capable of having a rational and conscious relationship with others and with Nature.

\subsection{Questions}

1. Do human beings meet boundaries in the ontology within their reach by cognitive structures associated to the information perceived in their close relationship with the fundamental laws of life?

2. If biology limits human's ontological boundaries, how are the design goals people use affected?

3. In a scenario of severe environmental deterioration, what design approaches would work? What would be the design requirements?

\section{LIFE'S LAWS, THE HUMAN BEING AND DESIGN}

It is important to differentiate between two questions, what is life? and what is life for? For it is possible to answer the first question, while solving the latter falls outside the realm of this document. In that light, it can be said that life is the process in which a finite amount of matter self-organizes, metabolizes (uses energy from the environment), grows, reproduces and evolves [1].

\subsection{Self-organization, metabolism and growth}

According to the second law of thermodynamics, life generates higher levels of order [2] by importing negative entropy for itself, also known as negentropy or syntropy, while exporting a larger amount of entropy to the environment. In the aforementioned thermodynamic exchange, life increases order in a process called data exchange [3]. This way, energy obtained from the environment is used through metabolism to organize molecules in coherent sequences of information, known as genetic information. The by-product of this process is heat release or the exchange of entropy to the environment.

Generation of higher levels of order or information exchange, require energy use and dissipation of heat. In this process, the cells of all living organisms are geared to increase the successful occupation of an ecological niche with a minimum of energy expenditure and seem to be bound by the laws of mass and energy transfer at a microscopic scale since all of them have very large surface to volume ratios in order to minimize local concentration and temperature gradients. Also, large multi-cellular organisms need vascular systems in order to distribute nutrients and oxygen in a very efficient way. This has been reflected in a universal mathematical rule called Kleiber's Allometric Law of Scaling [4]. 


\subsection{Reproduction}

Living beings deteriorate over time and, with deterioration, life eventually disappears. Nonetheless, the phenomenon of life, through the genetic mechanisms of reproduction, inherits an updated copy of its genetic information to a new being whose entropy is low and thus this enables the continuity of the process of life. When defining the guidelines by which life operates, the first one is that in spite of being circumscribed to the universal laws of physics, life uses everything within its reach to be continuous through time.

In the reproduction process of living beings, two different strategies can be observed, explained under the 'r/K Selection Theory' [5].

Living beings oriented towards an ' $\mathrm{r}$ ' reproduction strategy exist in unstable environments and non-saturated ecological niches where high speed of reproduction is crucial for survival. The characteristic features on this strategy are low energy investment to the descendants and high fecundity, low body volume, early maturity, short-life expectancy and wide dispersal of the population. Rodents are a mammal example. Scientific literature points ' $r$ ' selected species as opportunists.

Living beings oriented towards a ' $\mathrm{K}$ ' reproduction strategy exist in stable environments and niches close to their maximum load capacity; their fecundity rate is low, energy investment from parents to descendants is high, maturity is slow and life expectancy is long. Big mammals are the main examples of species under $\mathrm{K}$ selection. Scientific literature points ' $\mathrm{K}$ ' selection species as balance species.

\subsection{Evolution}

The physical margins where life is possible seem small, but there is enough room within them to generate adaptation needs [6]. These are covered in the genetic traits that persist or get discarded through the reproduction process.

Regardless of the fact that the most popular interpretation on Darwin's proposition, 'survival of the fittest', means survival of the biggest, strongest, and fiercest, the most accepted interpretation on Darwin's work is that fitness should be understood as the selection of genetic features that guarantee, on the long-run, the survival of the best adapted.

As an example, it can be said that in similar environments, discounting energy availability, living beings with high metabolic rates will be better adapted when energy is abundant and, given that abundance, it may be necessary to compete in a numerous population. On the contrary, in an environment with low energy availability, living beings with low metabolic rates and greater willingness to collaborate will be more successful [7].

\subsection{Restriction and capacity}

Life is limited by nature's laws, but given the opportunity, it widens its capacities to accumulate negentropy and responds to environmental changes using the evolution mechanism. In this light, one may conclude that there are two working axes that determine the success or failure of the changes and their persistence through time.

\subsubsection{Restriction. The environment, the external}

The list of environmental variables that affect living beings is long, but the most bounding resource is energy. Be it autotroph living beings and solar energy, or heterotroph beings and their food. The environment in which living beings develop may be of low or high restriction; there is no way anyone 
can say that there is an unbounded environment since: in spatial terms, life shares a finite environment known as Earth and it is not possible for it to survive isolated in the average conditions present outside the planet.

\subsubsection{Capacity. What is of each, the intrinsic}

With life's existence, in spite of the higher transfer rate of entropy towards the universe, a greater order is produced in its operating range. On the long term and as a second directive of life: life works to widen its possibilities of information exchange or negentropy accumulation. At the limit, life will achieve as much order as the available energy and entropy level of the environment will allow.

The dependence on the environment and its changes produce exchanges among the time permanence directive versus the capacity to generate negentropy (negentropic capacity) directive. The latter prevents that the speed in which life spreads through the environment to be constant since changes in the environment and restriction levels, define the necessity to modulate the evolution process in order to guarantee permanence in time.

The acceleration on capacity growth requires larger amounts of energy, either due to an increase in population or to an increase in the complexity of the living beings that are part of the process. However, in spite of the irrevocable calling of life to generate as much order as possible, the speed to do it will vary in time as a function of the acceleration and deceleration of the capacity to generate negentropy.

\subsection{Metabolic model}

This paper has described the features of living beings, biological and physical laws that limit them and two axes of action, restriction and capacity. In that light, and using the environment as the external and capacity as the internal or what is intrinsic to life, it is possible to make a schematic analysis of the gathered elements in a metabolic model generated by the intersections (strategies) of each category (Fig. 1).

Quadrant 1: Low restriction/Low capacity: Status Quo. On these coordinates, available resources supplied by the environment surpass the metabolic needs of the population. The environment is unstable either by reproductive competition and/or environmental variations. Additionally, there is no change in the capacity to generate negentropy. The reproductive rate is maximized but given the low negentropic capacity of the population, the resources consumption rate is lower than the environment's replacement rate.

Quadrant 2: High restriction/Low capacity: Survival. On this quadrant, the energy availability is compromised and there are no chances for expansion due to the impossibility to increase negentropic capacity. In metabolic terms, all energetic non-essential pathways disappear and energy use rate is minimized. If the environment's replacement rate is over-run, there is a reduction of population or death.

Quadrant 3: High restriction/High capacity: Transformation. The shortage of resources is compensated with an accelerated growth in negentropic capacity to take on new opportunities. There are potential but not present threats; every new function is enabled to seize a new opportunity within the environment.

Quadrant 4: Low restriction/High capacity: Growth. High availability of resources allows the colonization of the environment. It is possible to increase the negentropic capacity and the metabolic rate at the same time. Competition is produced by population's increase and metabolisms that consume more energy are favoured. 


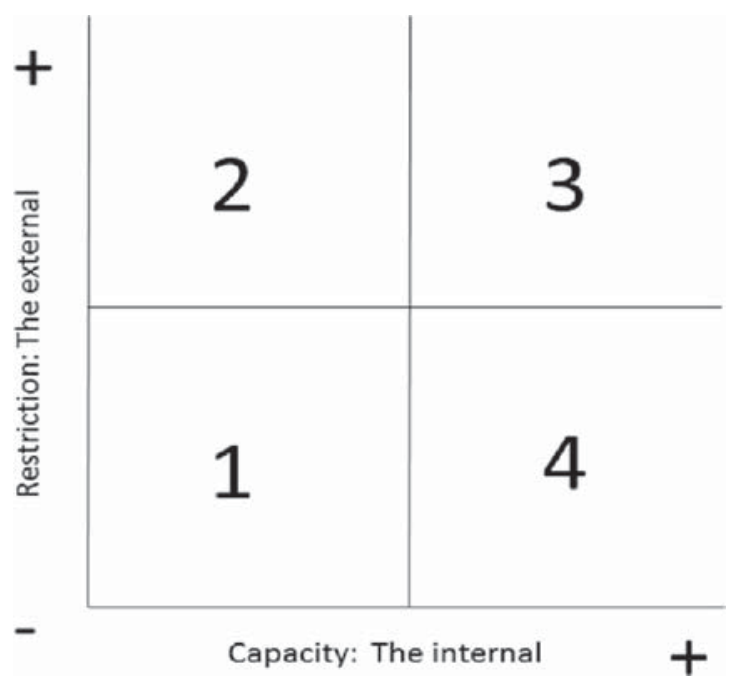

Figure 1: Schematic analysis.

\subsection{Hemispherical analysis of the model}

By dividing the plane into four sections with two lines one vertical and another horizontal, the resulting hemispheres will be: vertical cut, left and right hemispheres; horizontal cut, top and bottom hemispheres.

\subsubsection{Vertical}

Left: Low evolution and capacity. Environmental conditions overcome life's long-term evolutionary dynamics, options are limited. Negentropy generation is limited.

Right: High evolution and capacity. The long-term dynamic for capacity growth, works unbounded in any environmental conditions. Negentropy's generation grows, as well as energy use and the exchanged entropy with the environment.

\subsubsection{Horizontal}

Top: There is little waste of energy, cooperation prevails over competition. The challenge imposed by the environment is more important than rivalry, thereby metabolism is optimized. In addition, efficiency levels used to incorporate energy to life process are increased. On the reproductive side, selection is oriented towards ' $\mathrm{K}$ '; there is low variation in environmental factors and low rivalry level.

Bottom: Energy use is maximized; competition prevails over cooperation; reproductive competition is more important than the environment's challenge. Efficiency is not important, but final metabolic capacity is. Reproductive selection is oriented towards ' $r$ '; there is instability due to environmental factors and/or because a high degree of rivalry.

\subsection{Ecological niches}

To enhance the analysis it is also important to make reference to different types of living beings and the ecological niches they hold within the general model while explaining Fig. 2. 


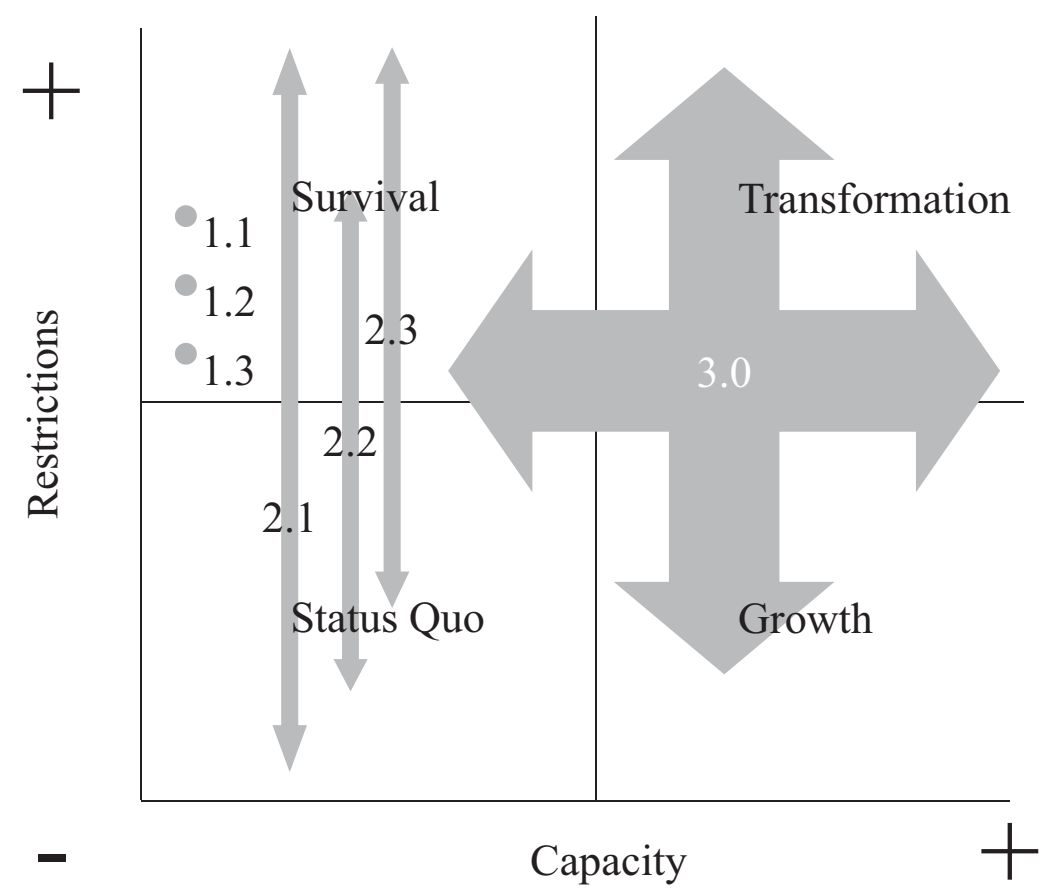

Figure 2: Ecological niches.

\subsubsection{Autotroph}

They populate the different restriction levels by differentiation of species, there is no locomotion or behaviour, in Fig. 2 a species is a point, the whole forms a negentropic capacity line (photosynthesis). They evolve through physical features or 'hardware' (Points: 1.1, 1.2, 1.3 in Fig. 2).

\subsubsection{Non-human heterotrophs}

They acquire the capacity so that each species populates a vertical line and, through locomotion, they generate a new adaptation strategy: migration, which means capacity to run away from danger, attack, pursue pleasure (instinct); behaviour emerges and is always closely related to 'hardware' configuration. Species manage to settle on new capacity spaces by differentiating their energy strategy: herbivorous, carnivorous, and omnivorous. They also evolve through 'hardware'. Locomotion accelerates evolutionary competition (lines $2.1,2.2,2.3$ in Fig. 2).

\subsubsection{Human heterotrophs}

They abandon evolution by 'hardware' (physical features) and represent a new evolutionary strategy for life, evolution through 'software'. They are capable to choose any quadrant, be it by migration, aversion to pain, attraction to pleasure and by cultural development. They have the ability to choose their behaviour, which adds extra capacity mechanisms and information to the ones granted by genetic inheritance. They manage to change the pattern resource usage in a quick way through a new mechanism, cultural evolution [8], that is inherited to their descendants through knowledge transmission (cultural reproduction) which means the accumulation of negentropy in culture (arrows 3.0 in Fig. 2). 


\subsection{Cultural evolution}

In biological terms, the human being is close to the extreme of ' $\mathrm{K}$ ' selection within the reproduction strategy. This means that it improves his descendants' survival possibilities by investing in them a great amount of energy. ' $\mathrm{K}$ ' selection also means there is a long maturity period for individuals and a longer life span.

Unlike some species under ' $\mathrm{K}$ ' selection, such as elephants and whales, the human being does not have great body volume or exceptional physical capacities, but it consumes huge amounts of energy. As stated before, the main difference on man's survival strategy lies within the reduction of physical and genetic competition with other species, in order to evolve quickly through a new strategy already defined as cultural evolution.

Exactly like genetic evolution, cultural evolution generates negentropy through the use of energy exchanged for higher levels of information expressed in knowledge, art and technology. Furthermore, just as it happens on genetic evolution, new levels of information (knowledge) generate new possibilities to access energy sources and for new ways to make use of the environment (technology). The latter is done in a way similar to what happened when energy strategies like the herbivorous, the carnivorous, and the omnivorous appeared. In Philosophy, a rise in the understanding of the universe is defined as an increase in the consciousness level or approximation to truth. In biology, the difference between conscious (man) and unconscious beings (the others) is also pointed out.

Even though the theme of consciousness is one of the most debated points in religious Theology and secular Philosophy, both approaches point at the need to answer the main ontological and cosmological questions: What exists? What is life for? Is the universe infinite? Does time have a beginning and an end? What is the essence of matter? It may be presumed that whoever manages to obtain an answer to any of this questions would be on a higher level of consciousness that those who don't.

Making definitions based on features that separate unconscious animals from conscious man has become complicated since Ethology reports more complex behaviours in animals than the ones assumed before (i.e. chimpanzee's use of tools, cooperation actions, learning and problem solving within various species), while neurosciences acquire better understanding of the human brain and report higher intervention of the subconscious in people's actions and motives than ever imagined [9].

Therefore, it is possible to say that the gradient going from the inert to the subconscious and to the conscious, is related to the non living, life and life's level of organization according to the capacity to generate negentropy. These positively correlates energy use and the level of consciousness, where the effective use of energy (energy efficiency), is more important than total use of it.

\subsection{Energy use and human development}

Figure 3 shows the relationship for 126 countries, between the Human Development Index (HDI) [10] and per capita energy consumption ( $\mathrm{ToE}=$ Tons of oil Equivalent) and the best adjusted curve seems to be a logarithmic model. It is worth noticing that all countries with HDI value higher than 0.9 use more than 2 ToE per capita. Also, all countries with per capita consumption lower than 0.7 ToE have an average HDI of 0.6. The corresponding empirical equation can be written as follows:

$$
\mathrm{HDI}=\mathrm{k} \ln \left(\mathrm{ToE} / \mathrm{ToE}_{0}\right)+\mathrm{B}
$$

Expression (1) has a threshold value when $\mathrm{ToE}=\mathrm{ToE}_{0}=1$, and, $\mathrm{HDI}=\mathrm{B}=0.737$, beyond which, the values of ToE increase very fast in order to approach the highest level of human welfare with per capita values of ToE higher than 3 . The value of the empirical constant, $k=0.1156$, seems to be a 


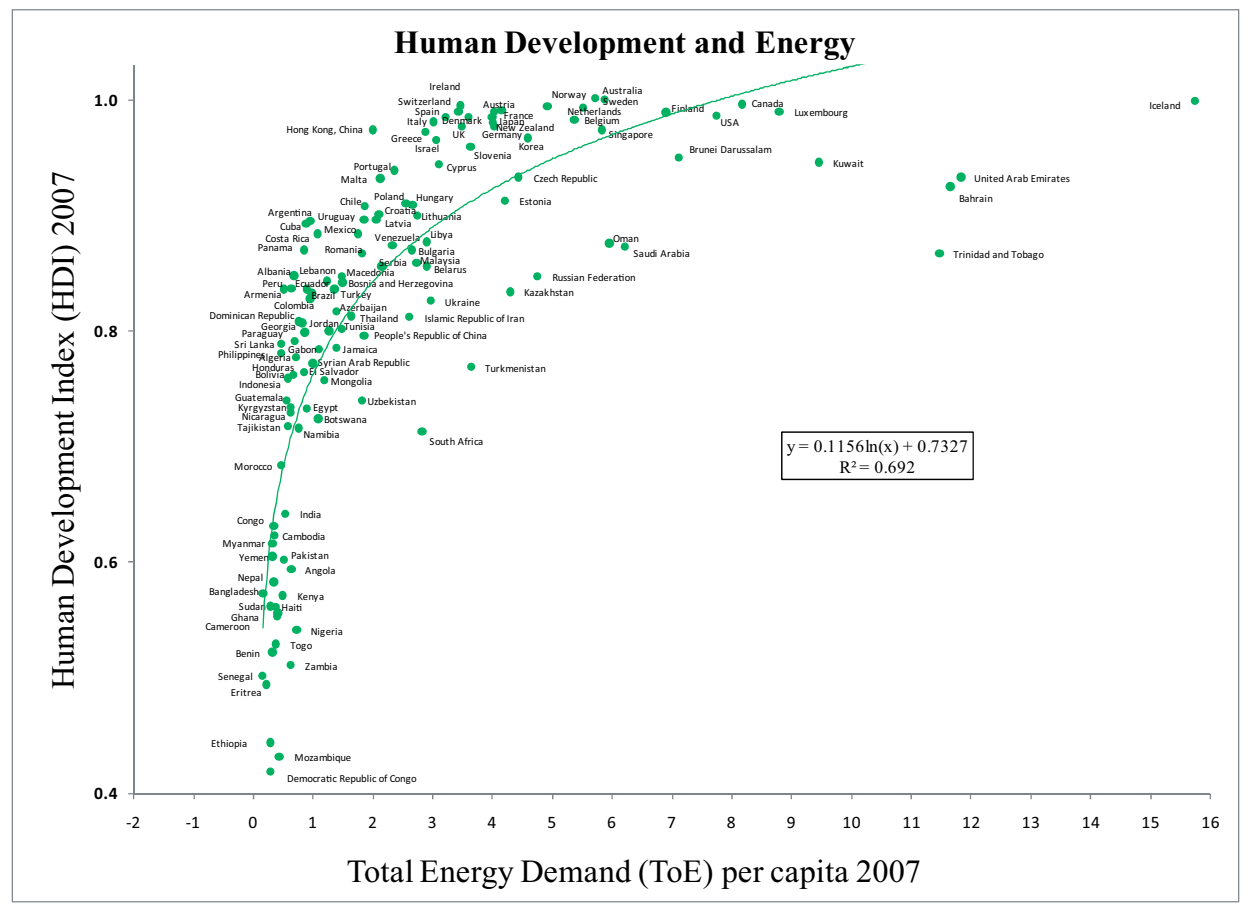

Figure 3: Human development and energy demand. Source: HDI, United Nations Development Programme (www.undp.org), Total Energy Demand, International Energy Agency (www. iea.org). ToE, Standardized unit equal to the amount of energy obtained through the combustion of a ton of crude oil. It is equal to: $41.868 \mathrm{GJ}$ and to $11.63 \mathrm{MW} / \mathrm{h}$.

global efficiency factor, since the correlation shown in Fig. 3 was taken from a sample of 126 countries (more than $90 \%$ of human population) and would be related to an intrinsic property of present human societies.

This is a very important consideration in terms of planning the future because it implies that the improvement of the quality of life for nearly 5 billion people, living with HDI lower than 0.7 , will require a very large increase of global energy expenditure. Otherwise, the social and political tensions across the world will increase breaking down the present level of international agreements. For example, terrorism and widespread violence will be difficult to eradicate in a world where most of the people experience a very low HDI environment.

\subsection{Energy use and technology development}

We can state that total energy use of a society $\mathrm{E}_{\mathrm{T}}$ has three terms

$$
\mathrm{E}_{\mathrm{T}}=\mathrm{E}_{\mathrm{M}}+\mathrm{E}_{\mathrm{G}}+\mathrm{E}_{\mathrm{RD}}
$$

where $E_{M}$ is the energy required for maintenance, $E_{G}$ is the energy required for growth and $E_{R D}$ is the energy to be invested in $R \& D$ (increase capacity). Here it is not intended to estimate each fraction of the total energy budget. Instead it is assumed that there should be a power law between $E_{R D}$ 
and $\mathrm{E}_{\mathrm{T}}$ and we also assume that energy expenditure is a reflection of the technological capacity of each society, measured as a normalized innovation index, $\mathrm{I}_{\mathrm{N}}$, defined as the number of patents registered in the U. S. Patent Office each year and assigned to nationals of each given country and normalized per million people. This index has two interesting properties, it involves the largest patent registrar in the world where international companies compete for the largest share in the US market, and also, it only takes into account the patents where the assignee (the owner of the patent rights) is from a given country. This way, the innovation index is strongly related to innovation supported by companies or individuals that wish to risk money in patent registration expenditures and have residence in a given country. The power law is of the following form:

$$
\mathrm{E}_{\mathrm{T}}=\mathrm{E}_{\mathrm{T} 0}\left(\mathrm{I}_{\mathrm{N}} / \mathrm{I}_{\mathrm{N} 0}\right)^{\mathrm{n}}
$$

Figure 4 shows the log-log correlation implied in the power law between $\mathrm{ToE}$ and $\mathrm{I}_{\mathrm{N}}$, it should be noted that from the previous list of 126 countries, only 74 have patents assigned to individuals or companies residents in those countries. It is worth noticing that the variation coefficient is only $\mathrm{R}^{2}=0.51$, suggesting that the total energy expenditures related to technological development require a more careful partitioning. But, it shows a general trend that may help to establish worldwide country typology in the way that energy expenditures are related to innovation. This preliminary model of energy expenditure follows the empirical equation:

$$
\mathrm{ToE}=2.1394 \mathrm{I}_{\mathrm{N}}^{0.2108}
$$

In Fig. 4, it can also be seen that the variable defined to evaluate capacity (patents/million inhabitants) increases through various magnitude orders that range from less than 0.1 patents a year per million inhabitants, up to more than 200 . This way, developing countries are clearly separated from developed ones. It is worth noting that there is not a single case of a developing country with low energy consumption and high patents' registration rate.

Again this seems to indicate the existence of threshold around $\mathrm{I}_{\mathrm{N}}=3$ patents per million people, as a requisite to reach an energy expenditure, per capita, of 3 ToE. In Fig. 4, there is shadowed region that covers from 3 to 10 patents a year per million inhabitants and seems to be the transition zone between development scales and contains countries under change process, from underdevelopment to development.

The slope of the empirical power equation in Fig. 4 may be used as an indication of the comparative efficiency reciprocal interaction between energy expenditures and technological development. There are some interesting cases to discuss. For example, the United Arab Emirates, Trinidad and Tobago, several oil exporter countries and some ex Soviet countries, have a value of $\mathrm{I}_{\mathrm{N}}$ lower than 0.33 patents per million although they have energy expenditures higher than 3 ToE per capita. However, they cannot be considered members of the highly developed nations club since their HDI is lower than 0.9.

The most suggestive aspects in Fig. 4 are the empty spaces at the right bottom and left top quadrants. Empty spaces indicate possible hindrances or forbidden zones, top and bottom, of a scaling rule between energy use and development. In fact when all points $(2,115)$ from a data base with a data set from 1978 to 2007 were plotted (Fig. 5) and analyzed with a potential model, the result was a regression equation equal to $\mathrm{y}=1.63 \mathrm{x}^{0.26}$, with a variation coefficient $\mathrm{R}^{2}=0.59$, where ' $y$ ' is equal to primary energy supply in ToE units and ' $x$ ' is equal to patents registered in year per million inhabitants.

After making a diachronic (related to time) analysis with an online tool named 'Motion Chart' from 'Google Docs', it was observed that the nation with best performance, and the only one that advances several orders of magnitude in both axes is South Korea, a nation that frequently is referred 


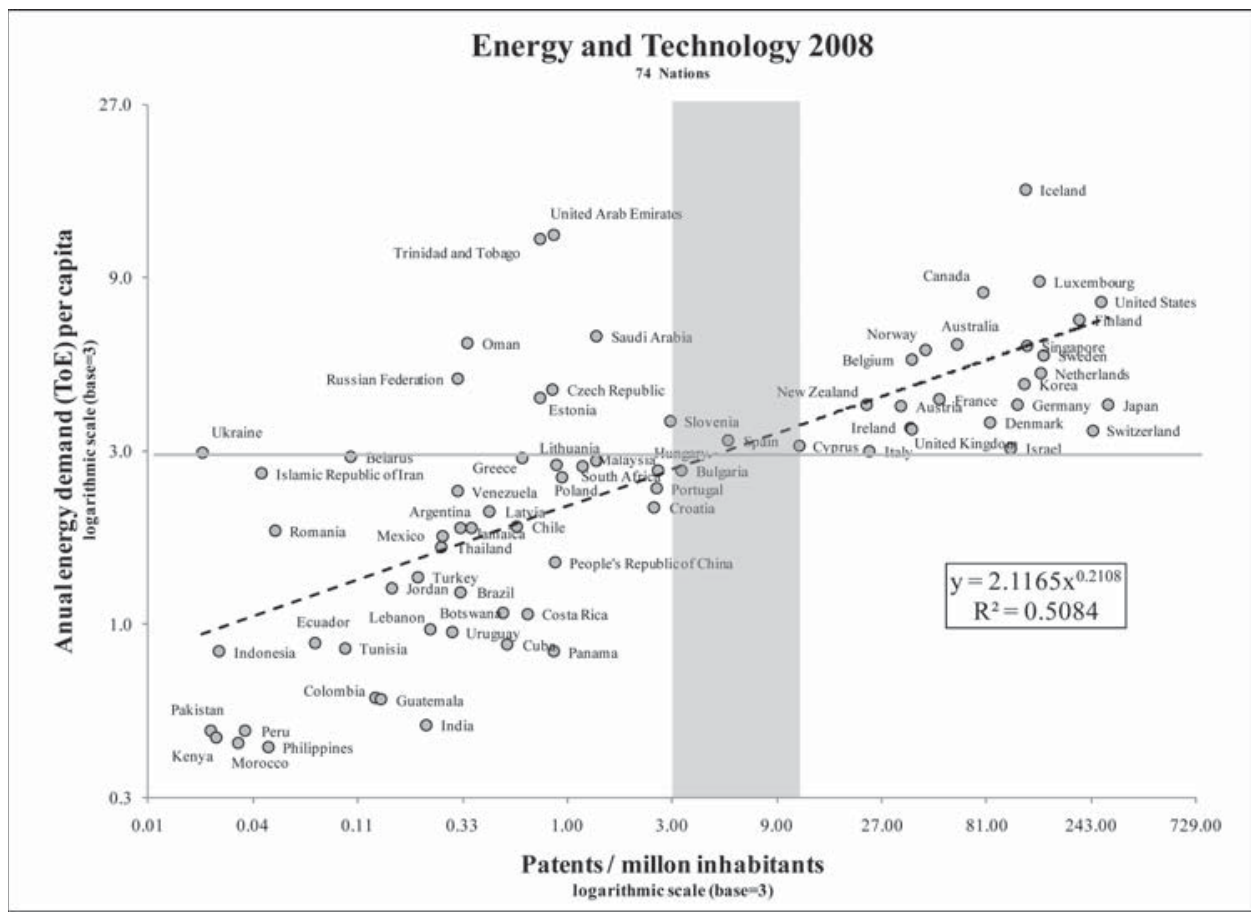

Figure 4: Energy and technology. Source: Energy International Energy Agency (www.iea.org) and patents from U.S. Patent Office (www.uspto.gov).

in specialized publications as an example of success given the fast achievement in economic and social development.

For this reason, a specific analysis was made only with South Korea's data, also presented in Fig. 5, here the set of couples of values $\left(\mathrm{ToE}, \mathrm{I}_{\mathrm{N}}\right)$ were plotted vs. time and the regression analysis showed the following empirical equation:

$$
\mathrm{ToE}=(1.45) \mathrm{I}_{\mathrm{N}}^{0.24},\left(\mathrm{R}^{2}=0.98\right)
$$

This is a striking result suggesting that this equation is indeed a scale-up equation between energy and technology and supports the notion that the power law of Figs 4 and 5 is the 'developmental pathway' for social and economic improvement. Here, the scaling coefficient is approximately ' $1 / 4$ ' .

Figure 6 shows the diacronic analysis of ToE vs. $\mathrm{I}_{\mathrm{N}}$ for Costa Rica, Mexico, South Korea and Saudi Arabia. The only growing up curve belongs to South Korea. The other three cases oscillated as if they were caught in an under development trap. Curiously enough the main numerical difference of those plots is not the slope (the power $1 / 4$ ) but the scale-up reference value $\mathrm{ToE}_{0}$.

Apparently, this is related to a given choice of resource allocation. A high level of $\operatorname{ToE}_{0}(>1.45)$ would imply to much attention paid to energy expenditure with low level of technology development, as seems to be the case of Saudi Arabia. A low level of $\mathrm{ToE}_{0}$ would imply that there is a weak coupling between technological development and energy expenditures. And there is a third case, Mexico where, here the trajectory is apparently superimposed to the ascending trajectory of South 


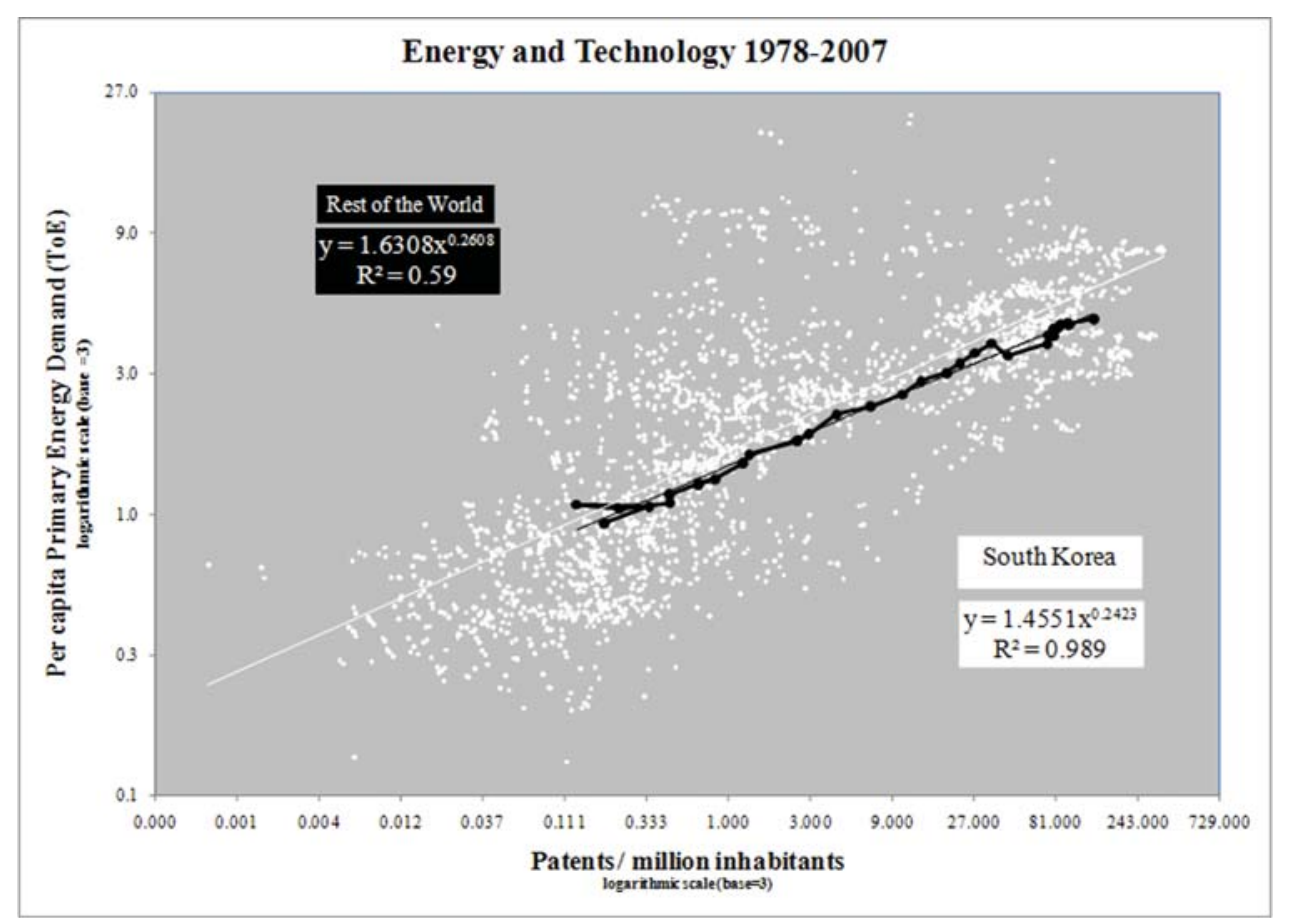

Figure 5: Evolution of energy and technology from 1978 to 2007. South Korean performance is indicated in the black curve with a correlation function $y=1.4551 \mathrm{x}^{0.2423}\left(\mathrm{R}^{2}=0.989\right)$. Source: Energy International Energy Agency (www.iea.org) and patents from U.S. Patent Office (www.uspto.gov).

Korea but in fact has oscillated in approximately the same place of the phase plot during the last 30 years. This also may be related to the lack of long-term commitment in order to establish a working link between technological development and energy expenditures. It is worth noticing that Mexican R\&D expenditures have been maintained at a level lower than $0.4 \%$ of GDP during the last three decades and the gap between scientific publication and technological innovation is well known and currently discussed during that period.

It is also worth noticing that the concepts 'economy of scale', and 'marginal analysis' used in Economics, come from the same principle as the biologic scaling rules: large systems are more efficient, but the costs they generate, including externalities - at some stage - grow faster than the benefits they generate, to the point where obtained additional benefit is lower than the cost increase. An example of probable boundaries and efficiency differentials is observed on the position held by Switzerland and the United States (Table 1).

To conclude this section, it is necessary to highlight that in a closed system like the earth, the noninclusion of externalities to the overall development cost accumulates systemic risks in the form of a reduction of the biospheres capacity to regenerate resources. In thermodynamic and biological terms, the accumulation of externalities is the accumulation of entropy, which means the consecution of the life expansion directive without balance with the permanence in time directive. 


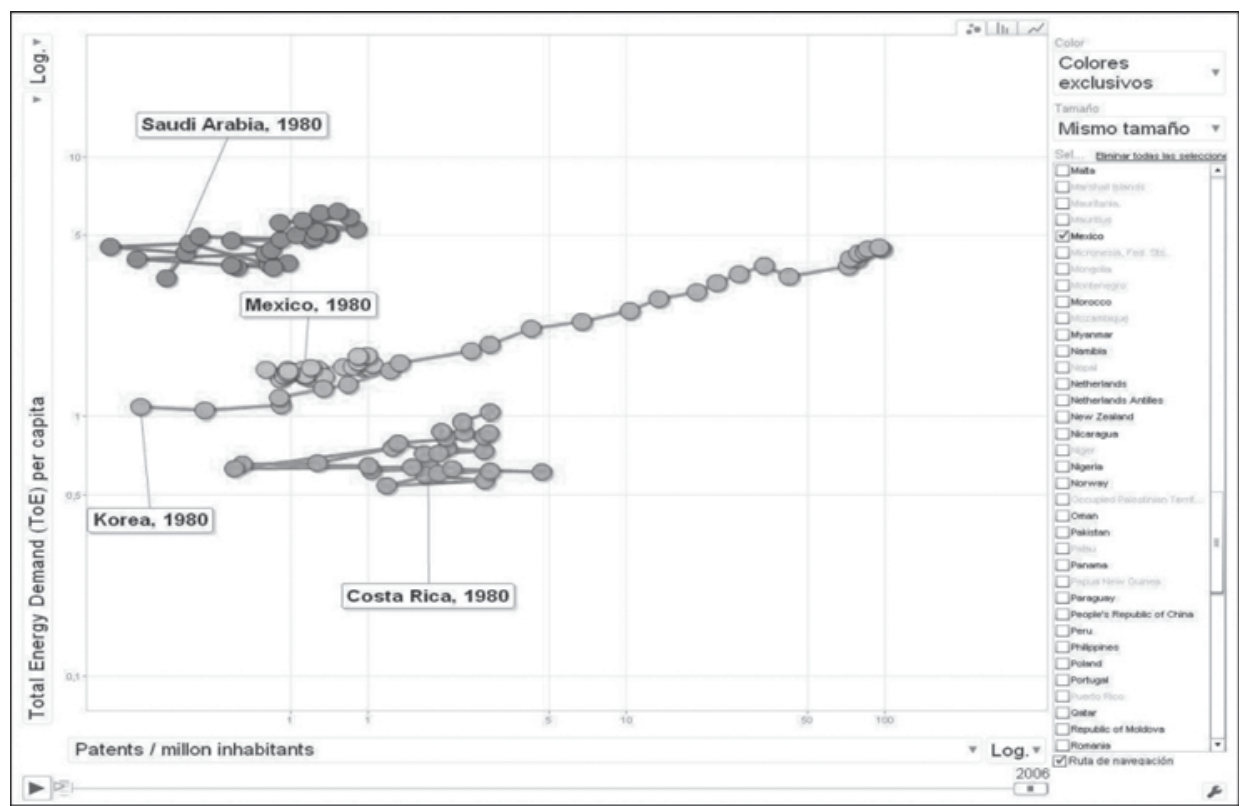

Figure 6: Diachronic analysis (selected countries). Diachronic analysis was made with 'Motion Chart' from Google ${ }^{\circledR}$ Docs (docs.google.com).

Table 1: Energy efficiency of Switzerland and USA.

\begin{tabular}{lcc}
\hline Concept & Switzerland & United States of America \\
\hline GDP per capita (USD) & 64,011 & 46,716 \\
Energy demand (ToE) per capita & 3.42 & 7.74 \\
Patents a year/million inhabitants & 246.07 & 267.61 \\
Human development index & 0.960 & 0.956 \\
\hline
\end{tabular}

\subsection{Design}

At present, the words design and evolution are used to frame a metaphysical debate between intelligent design and random evolution. This document sets aside the metaphysical debate related to the design and focuses on its physical aspect. In other words, it works on the mechanisms that affect the process through which human intelligence is mixed with the surrounding environment.

Although design is intuitively related to the liberal arts', manmade culture comes from creative acts. Every creative act, be it philosophical, artistic or industrial, is designed and behind any design effort is a design approach through which, the designer, gathers his capacities: ideas, techniques and desires around restrictions: physical laws, resources, space and energy availability.

Hence, restriction, capacity and philosophy are coordinated through design and even in our time it remains an intuitive process that comes more from the subconscious than from reason and science. For people, the first design approach rises from their conscious or unconscious answers to the questions in Table 2.

Around the internal world questions lie the main epistemological issue, courage to know the world. Since knowledge requires an increase in capacity to accumulate negentropy, and this is a 
Table 2: Design objectives questions.

\begin{tabular}{lll}
\hline Type of questions & \multicolumn{1}{c}{ Internal world } & \multicolumn{1}{c}{ External world } \\
\hline 1. Origin questions & What are my capacities? & What restrictions am I facing? \\
2. Destiny questions & Can I increase my capacity? & Can I overcome restriction? \\
\hline
\end{tabular}

costly process in terms of resources, time and effort, some people may have reasons to give up the increase of capacity.

With possible answers to questions in Table 2, people choose a design goal with which they move or stay at Status Quo, Survival, Transformation, or Growth. The choice of each design goal implies adopting a set of positions according to their individual assessment of their own capacities and reacting to the perceived restrictions presented by the environment.

The set of choices around capacity evaluation will be named 'Submission' for trying to stay or move into a low capacity scenario and 'Freedom' for moving or staying in a high capacity situation. In a similar fashion staying or moving into a high restriction situation will mean choosing an 'Optimization' position and doing so for the low restriction position will mean adopting a 'Maximization' strategy.

\subsubsection{Optimization versus maximization}

It has been established on the horizontal dimension of the model's hemispherical analysis that, in metabolic terms, living beings function as follows:

Up (optimization): There is low environmental instability where cooperation prevails over competition; an efficient metabolic arrangement is more important than total metabolic capacity. This design vector will be named 'Optimization', where system variables are arranged to obtain the best result in systemic terms. This is consistent with pursuing: empathy, planning, Ethos, adherence to external law, efficiency, low externality, cooperation, virtue, and common good.

Down (maximization): There is high environmental instability and competition prevails over cooperation while reproductive competition and total metabolic capacity is more important. This design vector will be named 'Maximization'. In it, system variables are subdued to obtain the highest performance of a reference variable. This is consistent with pursuing: apathy, reaction, competition, no Ethos, waste, externality, own law, self-interest, and vice (Fig. 7).

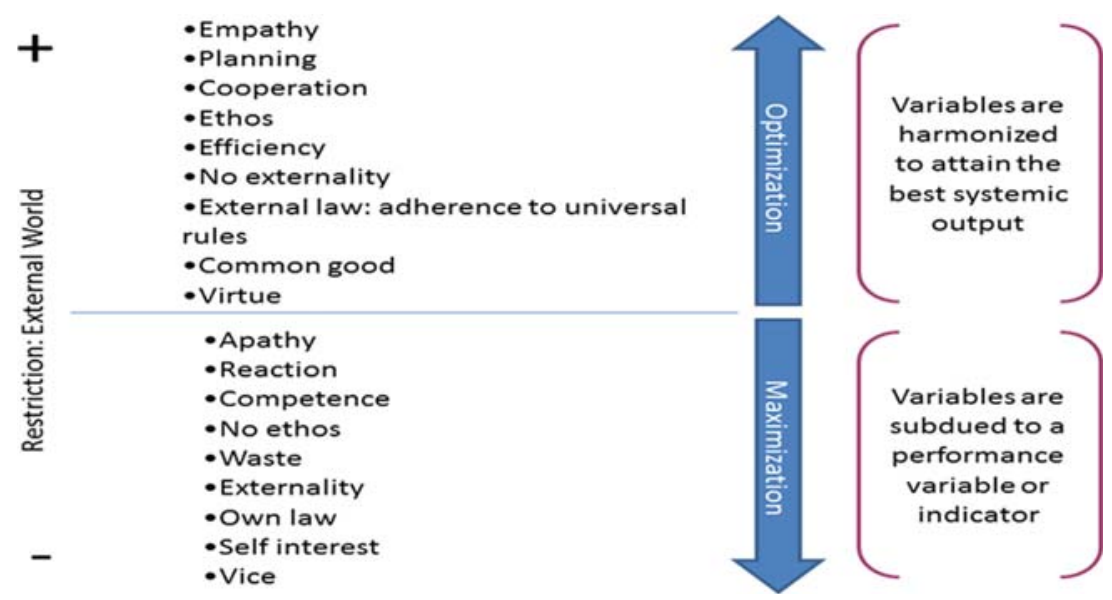

Figure 7: Optimization versus maximization. 


\subsubsection{Submission versus freedom}

Regarding the vertical dimension of the hemispherical analysis, the following was established:

Left (submission): Low capacities to generate negentropy, options are limited. This design vector will be named 'Submission'. Given the low negentropic capacity, the energetic cost to generate an increase in capacity represents an expensive bet in terms of exchange of strategy. Additionally, more value is given to what a person has, than to what can be obtained; this means inability to exchange strategy and derives in: ignorance, disease, acceptance of time/life reduction, low energy use, insecurity, guilt, change and risk reluctance, narrow-mindedness, restrictions.

Right (freedom): High capacity to generate negentropy creates a wide range of options. The data exchange grows and so does energy use and exchange of entropy with the environment. This design vector will be named 'Freedom'. Given the high negentropic capacity, it is easy to make energy investments destined to explore new possibilities. Moreover, it means freedom to evolve according to: knowledge (Logos), health, time/life increase, higher energy use, security, responsibility, change and risk acceptance, openness, ideals (Fig. 8).
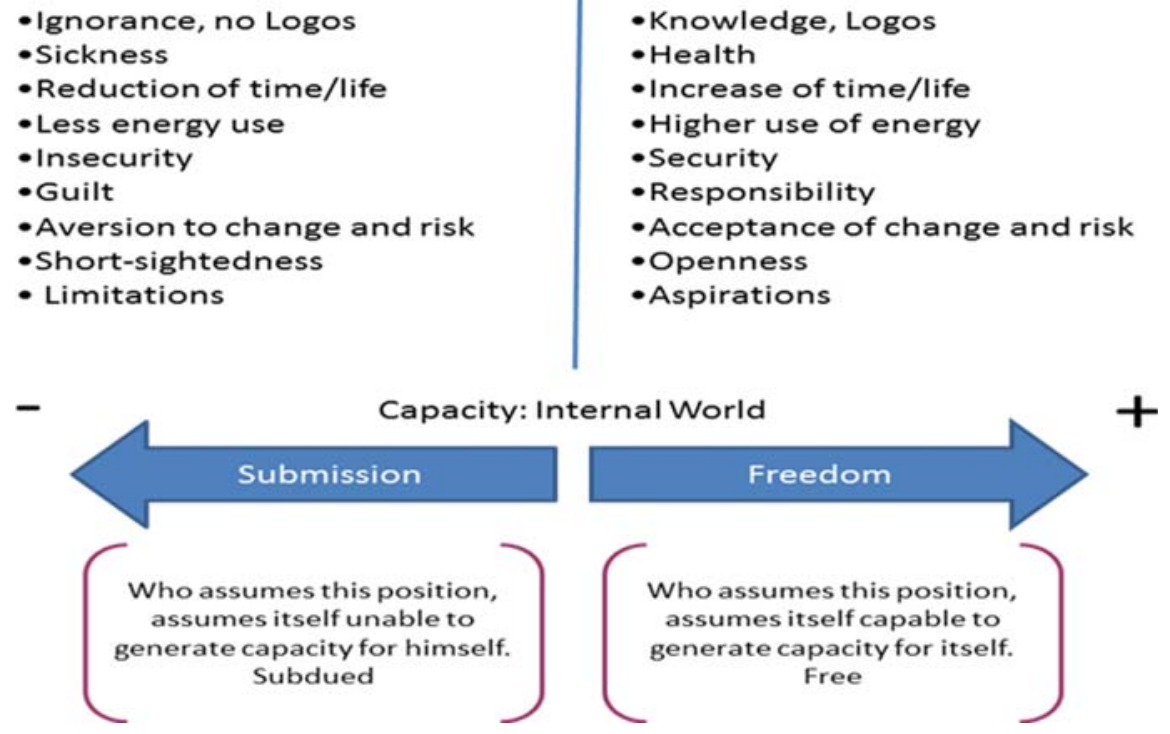

Figure 8: Submission versus freedom.

\subsection{Evolution of configuration}

Because human culture is part of the evolutionary process, it is necessary to distinguish design from evolution of configuration. At present, the great cultural dilemma circles around what political configuration should a society use. The discussion is focused on two base possibilities [11]:

- Closed society. Within low capacity environments (submission), mechanisms to which people are subdued are favoured. This is consistent with the idea that when people give up capacity growth, 
the environment becomes more dominant over people's behaviours and choices, and they expect the existence of an authority that designs and re-designs the rules.

- Open society. In high capacity (freedom) environments, emerging mechanisms are favoured, where there are few rules and wide operating margins. People expect minimum interference from authority and participation in designing and redesigning the rules.

Earth's inhabitants are under the influence of some authority regime, represented by NationStates; so it is hard to separate design and regulation, since modern States use Law to code design and regulation (configuration) with a single tool.

Since the same mechanism is used to make social design and to establish regulation, an identity is created: 'Law is design and design is regulation'. The latter is deeply embedded in modern conception of cultural design. As it was mentioned at the beginning of this chapter, design is the coordination of possibilities and restrictions. In other words, once the design is established, it becomes the frame where a set of interactions can take place, and regulation is the configuration of the design arrangement.

It has been said that a human being chooses between capacity growth (freedom) or giving up that possibility (submission), so configuration evolution may be found among two possibilities:

- 'Bottom Up': When individual's subordination to an authority and regulations focused to determine his behaviour are low and change speed and trust [12] are high. This is a participative process in which authority and society concur.

- 'Top Down': When individual's subordination to an authority and regulations focused to determine his behaviour are high and change speed and trust are low. The process is non inclusive, only the authority participates.

In human culture, unlike biological and physical systems, the plasticity that self-determination of action of people, allows redesigning even at configuration and evaluation levels.

According to this, it must be realized that 'Design' is a project activity; it has a purpose, a beginning and an end. '...A project is always a pro-iectum, something that is launched before us to leave the place we are in, to ascend...' [13]. 'Configuration evolution' is a process activity; it is constant and must be circumscribed to the boundaries of the 'Design'.

\subsection{Ontological design}

Now it is necessary to analyze whether the peculiar features of design and the proposed model are good to build the bridge that links the necessary elements to answer the question: 'Do human beings meet boundaries in the ontology within their reach by cognitive structures associated to the information perceived in their close relationship with the fundamental laws of life?'

The fundamental blocks with which Philosophy is built are:

- Metaphysics. Within Philosophy, it refers to the research that tries to give an answer to fundamental nature of the being and of the world; it is subdivided into Ontology and Cosmology.

- Epistemology. It refers to knowledge theory: What is known? How is it known? What is knowledge? Why is it known what is known?

- Ethics. It is the behaviour theory over which values that guide people's actions are determined.

- Politics. Process through which groups of people take decisions. Within the classical definition of Politics, Economics and Law are subordinate subsets of it. 
To discuss metaphysical selection options within the ontology's aspect, according to the rank of design goals previously established. 'Motivation Theory' [14] will be used to determine, what motivates people to act:

Extrinsic - when a person's motivation comes from the outside, it is the approach to 'Have' (fame, fortune, power). It corresponds to a 'Materialistic' metaphysical approach; what can be obtained from outside or from others.

Intrinsic - when a person's motivation comes from inside, it is the approach to 'Do' (own merits, satisfaction for a well done task, expertise, discovery). It corresponds to an 'Objectivist' metaphysical approach. What can be obtained on its own.

Transcendent - when a person's motivation comes from the outcome in someone else, it is the motivation to 'Be' (service, common good, unselfishness). It corresponds to a 'Transcendent' metaphysical approach; what can be generated by one's own existence.

By rearranging the proposed elements, Table 3 is obtained:

\subsection{From biology to philosophy}

The relationship between the laws of Biology, design goals and Philosophy takes place through the following process:

Living beings are found in any of four possible metabolic strategies due to the exchanges generated within the relationship between restriction and capacity.

Human being's inherent self-determination capacity allows them to choose among:

- Staying in the quadrant given at birth by cultural and genetic inheritance;

- Moving towards a different strategy according to their evaluation of capacity and restriction.

Derived from the decision a design goal is defined through the selection of the design vectors:

- Internal World: $\leftarrow$ Submission or $\rightarrow$ Freedom

- External World: $\uparrow$ Optimization or $\downarrow$ Maximization

Once the design goal has been chosen, the ontological approach over which the philosophy of life that will guide existence is adopted (Table 3).

Table 3: Building blocks of philosophy and design goals.

\begin{tabular}{|c|c|c|c|c|}
\hline & \multicolumn{4}{|c|}{ Design goals } \\
\hline Category & 1. Status Quo & 2. Survival & 3. Transformation & 4. Growth \\
\hline Ontology & Materialism & Determinism & Transcendent & Objectivism \\
\hline Motivation & To have & Renounce & To be & To do \\
\hline Restriction vector & Maximization & \multicolumn{2}{|c|}{ Optimization } & Maximization \\
\hline Ethics & Self-interest & \multicolumn{2}{|c|}{ Common good } & Self-interest \\
\hline Capacity vector & \multicolumn{2}{|c|}{ Submission } & \multicolumn{2}{|c|}{ Freedom } \\
\hline Epistemology & \multicolumn{2}{|c|}{ Superstition } & \multicolumn{2}{|c|}{ Reason } \\
\hline Politics & \multicolumn{2}{|c|}{ Closed } & \multicolumn{2}{|c|}{ Open } \\
\hline Configuration & \multicolumn{2}{|c|}{ Top down } & \multicolumn{2}{|c|}{ Bottom up } \\
\hline
\end{tabular}


This means that human beings find boundaries in the Ontology within their reach, due to cognitive constructions linked to information gathered in their close relationship with the fundamental laws of life.

\section{QUADRANTS, POLITICAL SYSTEMS CHARACTERIZATION AND TYPES OF DESIGN}

Although the formal characterization of a large group of societies requires both profound historical and statistical analyses, the evidence here presented allows for a simple, yet powerful, characterization of the systems and design objectives (Table 4).

Linked to what was said in the characterization of quadrant 'Transformation', we find the ideas explored by some of the main theological systems that aim into the pursuit of truth, freedom, perfection, and an ethical behaviour to reach the balance produced by virtue. Nonetheless, there is no political system design that contains a theory of man with which a design of transformational social technologies (economics and law) can be made possible.

Nevertheless, out of the research done for this paper, there are some fresh ideas for a 'Transformational' design based on a flow system arrangement. Although this is a subject not addressed in this article, it seems feasible to continue work for the description of what can be considered 'Metabolic Design', 'Metabolic Economics' or 'Constructal Economics'.

\subsection{Design goals and differences in social design}

Regarding the question: 'How are the design goals people use affected?' the proposed model allows an integral analysis comprised of four design approaches and the philosophies that align them with each design objective. The latter is made in connection with fundamental laws of life and enables us to size the depths that separate each of their practitioners.

Table 4: Characterization of political systems.

$\begin{gathered}\text { Survival: ancestral survival } \\ \text { cultures, such as Kenya's } \\ \text { bushmen and the }\end{gathered}$
$\begin{aligned} & \text { Amazon's Yanomami. } \\ & \text { Theological social design. }\end{aligned}$ $\begin{gathered}\begin{array}{c}\text { Transformation: there is no explicit political design for this } \\ \text { system. Post-industrial market economies' designs that keep } \\ \text { a balance between market and social cohesion systems, such } \\ \text { as those in Scandinavian nations and Japan, and whose } \\ \text { special feature is the persistence in modern culture of } \\ \text { concepts like 'Lagom' (from folk Swedish culture, not too } \\ \text { much, not too little), and 'Shinto' (Ancient cultural and } \\ \text { religious root from Japan centered in harmonic relationship } \\ \text { with nature and people), can be considered close to this type } \\ \text { of system. Transformational social design. } \\ \text { Growth: several of the western capitalist economies known as } \\ \text { developed countries, recently BRIC nations (Brazil, Russia, }\end{array} \\ \begin{array}{l}\text { India, China). Competitive social design. } \\ \text { Status Quo: authoritarian } \\ \text { systems such as: empires, } \\ \text { fiefs, dictatorships, } \\ \text { autarchic monarchies and } \\ \text { countries rich in natural } \\ \text { resources and poor in } \\ \text { human development. } \\ \text { Institutional social design. } \\ -\end{array}\end{gathered}$


Additionally, tensions produced by the interaction among groups that build social technologies from different ontological positions can be identified. Although in this paper strategy changes have not been addressed, research done on the subject, indicates that it will be more likely to start a change of strategy when a human group is self-perceived in a survival situation and once the possibility of change appears there are two alternatives:

- Revolution of capacities, getting out of the problem by developing higher capacity;

- Aggression, reducing restriction by appropriation of somebody else's resources.

It can also be stated that there is a third possibility of change when a society placed on quadrant ' 3 . Transformation' changes the optimization perspective for a maximization one.

\section{DESIGN GOALS AND ENVIRONMENTAL ADVERSITY}

Modern era's success on capacity growth is beyond dispute and, as proven by the reduction of authoritarianism at the end of the 20th century, an economic system that metabolizes resources as fast as capitalism does, in the long term, also allows us to think about an affluent and open society. However, evidence indicates that the economic metabolism generated by the human kind overcame, several decades ago, the recovery capacity of the biosphere, because less crop lands, fresh water, clean air, and energy are available, while needs increase due to population growth and cultural evolution [15].

In spite that around a billion people [16] have access to high levels of energy, health and knowledge, the rest of humanity, in decreasing gradients, live on higher restriction and knowledge environments (Fig. 3). It is from this difference that confrontation among people emerges and prospers in an increasing scale, whose foundations have been explained before.

At the end of the day, population projections [17] indicate that by year 2050 there will be eight to ten billion people over the earth. This means, by following current trends, that the return to low consumption models will only happen if either a reduction of population and/or rationing of resources by restoration of authoritarian regimes occur (follow the permanence directive). By that token, freedom of action generated by open systems would not be possible. In fact, famine and upheavals would provoke a favourable environment for a regression to authoritarianism first hand around low capacity contexts. It is worth retaking the statistical exercise shown in Fig. 4 to include the democracy index [18]. Table 5 is made by obtaining average value of the democracy index, grouped by energy scales.

Two outcomes, linked to the model, derive from the above. On one side, the idea that development, energy and freedom are related is strengthened. On the other side, it seems unlikely to keep the existence of open political systems when energy incorporation to people's lives through use of technology is low, as can be seen on scales one and two of Table 5, and neither when there is no balance between energy abundance and cultural development.

Due to the above, and even if new environmental and energy technologies can manage the current adverse horizon, remaining within the 'growth' quadrant will not be possible, given that sooner or later the energy restriction will be reached again. In Economics this never ending desire for something else once all needs are covered, is often referred as the eternal peddler problem, which also means finding uses for any new energy source, and the inevitable generation of a cycle among affluence and crisis.

In Economics, this recurrence is described as an economic cycle and it is important to point out that as the affluence stage is greater, crisis also become deeper and more destructive due to the risk accumulation on over scaled economic systems. Translated to a concept coined during the last world 
Table 5: Energy intensity and democracy.

\begin{tabular}{ccccccc}
\hline$\#$ & $\begin{array}{c}\text { Scale } \\
(\text { ToE })\end{array}$ & $\begin{array}{c}\text { Total } \\
\text { countries }\end{array}$ & $\begin{array}{c}\text { Democracy } \\
\text { index average }\end{array}$ & Regime type & $\begin{array}{c}\text { Example } \\
\text { countries } \\
\text { (democracy) }\end{array}$ & $\begin{array}{c}\text { Human } \\
\text { development } \\
\text { index average }\end{array}$ \\
\hline 1 & $0-1$ & 44 & 4.69 & Hybrid & $\begin{array}{l}\text { Ethiopia 4.72 } \\
\text { Burundi 4.51 }\end{array}$ & 0.632 \\
2 & $1.01-3$ & 28 & 6.35 & $\begin{array}{c}\text { Distorted } \\
\text { democracy }\end{array}$ & $\begin{array}{l}\text { Colombia 6.40 } \\
\text { Macedonia 6.33 }\end{array}$ & 0.823 \\
3 & $3.01-10$ & 31 & 7.75 & $\begin{array}{c}\text { Distorted } \\
\text { democracy }\end{array}$ & $\begin{array}{l}\text { Taiwan 7.82 } \\
\text { Estonia 7.74 }\end{array}$ & 0.930 \\
4 & $>10.01$ & 3 & 6.43 & $\begin{array}{c}\text { Distorted } \\
\text { democracy }\end{array}$ & $\begin{array}{l}\text { Filipinas 6.48 } \\
\text { Indonesia 6.41 } \\
\text { Distorted } \\
\text { Guatemala 6.07 }\end{array}$ & 0.903 \\
\hline
\end{tabular}

financial crisis, 'too big to fail' [19] is the inefficiency measure of a system and of the systemic risk that makes it unstable. This instability issue represents the main failure of contemporary economic systems. In biotechnology, there is a similar example where it has not been possible to overcome a barrier when genetically modified organisms used to maximize the return on products with economic value, obtained from harvests and fermentation, within few generations revert to a homeostatic genetic and metabolic condition.

Hence, when looking at Biology and Economics, the use of a maximization strategy, meaning the subordination of all systems' variables to a single result variable, will increase the probability of a crisis in direct relation to the gap measured against a stable evolutionary path. It can also be said that the ever increasing discovery of scaling laws, such as Kleiber's Law, represent the mathematical expression of the stable or persistent evolutionary paths complex systems follow.

Related to the later it can be said that life, in spite of existing in an inhospitable universe whose entropy grows continuously, tries through all possible means, to support the evolution process by rising negentropic capacity via an increase of conscience or accumulation of knowledge. And persist in time up to the limit that energy availability and entropy will allow. The same applies to the human being.

The reader should take into account that what was said above has implications for the 'What's life for?' question, which as stated at the beginning of section three, falls out of the scope of this work. It seems inevitable to try answer the question, while putting together life's two directives (survival and evolution), and assumption number three, 'Sensory experience makes possible the creation of concepts over which human cognition is built'. For this reason we will only add to this aspect of the discussion that the approaches for social design that seem more stable and functional are the ones that balance both the permanence in time and evolution directives. Although someone might try to get some sense of direction out of this, it cannot be said that purpose of life question has been answered.

\subsection{Functional design approaches}

When addressing the question: 'What design approaches would be functional?' we have that low capacity (survival and Status Quo) environments are temporary shelters when possibilities to 
increase capacity are low, or when freedom decrease or regression happens; within these strategies time can be earned, but capacity increase, evolution and freedom are given up, which happens in prejudice of attaining life's second directive (expansion). The growth environment is consistent with the directive to increase order generation and information, but high metabolic rates deplete resources faster than knowledge to acquire new capacity, so continuity probabilities diminish.

In consequence, the path where expansion and permanence in time are balanced is on the transformation quadrant. Nevertheless, that design goal has only been explored by Theology and lacks the political and philosophical designs that can allow modern man's cognitive transformation into post-modern man. In other words, the post-modern man will be the one who reconciles freedom with the universal laws that rule him.

To pretend that postmodernism can be invented by the man with a modern mind set, or that longs for Status Quo or a return to the original state, and even for the one who mixes design philosophies, is dissonant. All designs arise from an ontological position, so the first requirement is the existence of the post-modern man that designs the postmodernism in which he wants to live.

\section{CONCLUSION}

\subsection{Design boundaries}

Throughout this paper we have discussed the limits that define biological and cultural design, and now it is very useful to have a better understanding of the design rules present at nature, for life's processes as well as for non-living systems. Mathematical relations on the subject, such as the Fibonacci's series and the allometric laws of nature, have been known for centuries. However, this kind of phenomena has been collected as interesting occurrences of nature, until recent advances in science, such as the Constructal Theory [20], have gained insight into the universal laws of design. The Constructal Theory states that:

- 'For a finite-size (flow) system to persist in time (to live), its configuration must evolve such that it provides easier and easier access to its currents.'

- 'Every system is destined to remain imperfect (it will have restrictions), so it is necessary that the design of the system comes from the homogeneous distribution of the restriction.'

Important engineering progress has been achieved with the constructal design approach to develop systems with optimal performance in terms of persistence and efficiency [21]. Furthermore, now there is an explanation on the allometric laws that define the borders of biological design, such as the mentioned Kleiber's Law, which represents millions of years of evolutionary experimentation.

\subsection{Design requirements}

The last question to be answered to conclude this work is: what would be the design requirements? This document has provided an explanation on four design strategies, where for three out of the four possibilities, there are philosophies, political technologies and implementation experiences. However, there is a gap regarding the necessary political technology to build a world, or culture, or coexistence deal among people, within the transformational design objective. Hence, 
here are some design requirements for transformational (metabolic or constructal) social technologies:

In order to shape political technologies: (a) integrated, that cover law and economy; (b) evolutionary, free or with low regulation and (c) consistent with the transformation quadrant of the model, it is necessary that the design:

- Does not generate incentives to non-cooperation.

- Internalizes the cost of social and environmental externalities.

- Frees restrictions so that motives (ethics) and not incentives (instinct) become the dominant mechanism for social regulation.

- Reduces the distance on the capacity contexts in which people develop in order to decrease differences within the design objectives they use.

- Widens free flow of the information and knowledge streams.

- Produces a governance model by design and coordination and not government through regulation.

\section{REFERENCES}

[1] Nielsen, P., A new molecule of life. Scientific American, December 2008.

[2] Rubí, M., Does nature break the second law of thermodynamics? Scientific American, October 2008.

[3] Brillouin, L., Negentropy. Journal of Applied Physics, 24, pp. 1152-1163, 1953. doi:http:// dx.doi.org/10.1063/1.1721463

[4] Kleiber, M., Body size and metabolic rate. Physiological Reviews, 27, pp. 511-541, 1947.

[5] Pianka, E., On r and K selection. American Naturalist, 104, pp. 592-597, 1970. doi:http:// dx.doi.org/10.1086/282697

[6] Darwin, C., The Origin of Species, John Murray: London, 1859.

[7] Kropotkin, P., Mutual Aid: A Factor of Evolution, Forgotten Books: London, (Conclusion), 1902.

[8] Teilhard, P., The Phenomenon of Man, Evergreen Review: New York, p. 429, 2009 (e-book location).

[9] Zaltman, G., Marketing Metaphoria, Harvard Business Press: Boston, pp. 47-61, 2008.

[10] United Nations Development Programme (UNDP), www.undp.org

[11] Popper, K., The Open Society and Its Enemies, Routledge: London, 1945.

[12] Zak, P., The neurobiology of trust. Scientific American, pp. 62-67, June 2008.

[13] Llano, C., Función, Plan y Proyecto. Tópicos, 10, pp. 25-59, 1996.

[14] Pérez, J., Liderazgo y Ética en la Dirección de Empresas, Deusto: Madrid, 1998.

[15] Stern Review on the Economics of Climate Change; HM Treasury, U.K. Economic Service, www.hm-treasury.gov.uk/sternreview_index.htm

[16] World Population Estimate; United States Census Bureau, International Database, www.census.gov/ipc/www/idb/worldpopinfo.php

[17] United Nations, World Population Prospects. Department of Economic and Social Affairs, Population Division, United Nations. United Nations: NewYork, p. 12, 2008.

[18] The 2007 Index of Democracy, The Economist, Intelligence Unit, www.economist.com/media/pdf/democracy_index_2007_v3

[19] Watts, D., Too big to fail? How about too big to exist?. Harvard Business Review, p. 16, June 2009.

[20] Bejan, A. \& Lorente, S., Constructal theory of generation of configuration in nature and engineering. Journal of Applied Physics, 1004, 2006.

[21] Constructal Web Site, www.constructal.org 